\title{
Corela
}

Cognition, représentation, langage

HS-31 | 2020

Métalinguistiques.

\section{Sur une intonation à valeur métalinguistique}

\section{Evelyne Saunier}

\section{OpenEdition}

\section{Journals}

Édition électronique

URL : http://journals.openedition.org/corela/11622

DOI : 10.4000/corela. 11622

ISSN : 1638-573X

\section{Éditeur}

Cercle linguistique du Centre et de I'Ouest - CerLICO

\section{Référence électronique}

Evelyne Saunier, "Sur une intonation à valeur métalinguistique », Corela [En ligne], HS-31 | 2020, mis en ligne le 02 juillet 2020, consulté le 03 juillet 2020. URL : http://journals.openedition.org/corela/ 11622 ; DOI : https://doi.org/10.4000/corela.11622

Ce document a été généré automatiquement le 3 juillet 2020

\section{(c) (i) (2) (2)}

Corela - cognition, représentation, langage est mis à disposition selon les termes de la licence Creative Commons Attribution - Pas d'Utilisation Commerciale - Partage dans les Mêmes Conditions 4.0 International. 


\title{
Sur une intonation à valeur métalinguistique ${ }^{1}$
}

\author{
Evelyne Saunier
}

\section{Présentation}

1 L'intonation qui nous intéresse ici porte sur la reprise d'un dit $\mathrm{P}$ précédent, qui remet en cause l'adéquation de $\mathrm{P}$ à dire l'état de choses en jeu dans la conversation. Nous l'appellerons "Mise en doute métalinguistique" (désormais MDM). Il s'agit d'une intonation que l'on pourrait gloser par « $P$ ? c'est vite dit / il faut le dire vite/ je n'appellerais pas ça $P »$, et dont le contour est caractéristique. Nous l'illustrerons par le contexte construit ${ }^{2}$ suivant :

A. - Bonjour le binôme, ça va? Paul est toujours en vacances? Ça doit être tranquille, depuis qu'il est parti?

B. - Il est parti...

C. - Il a emmené tous les dossiers et il nous envoie trente mails par jour avec réponse urgente exigée ! On n'appelle pas ça partir, nous.

2 La courbe typique se présente ainsi ${ }^{3}$ : 


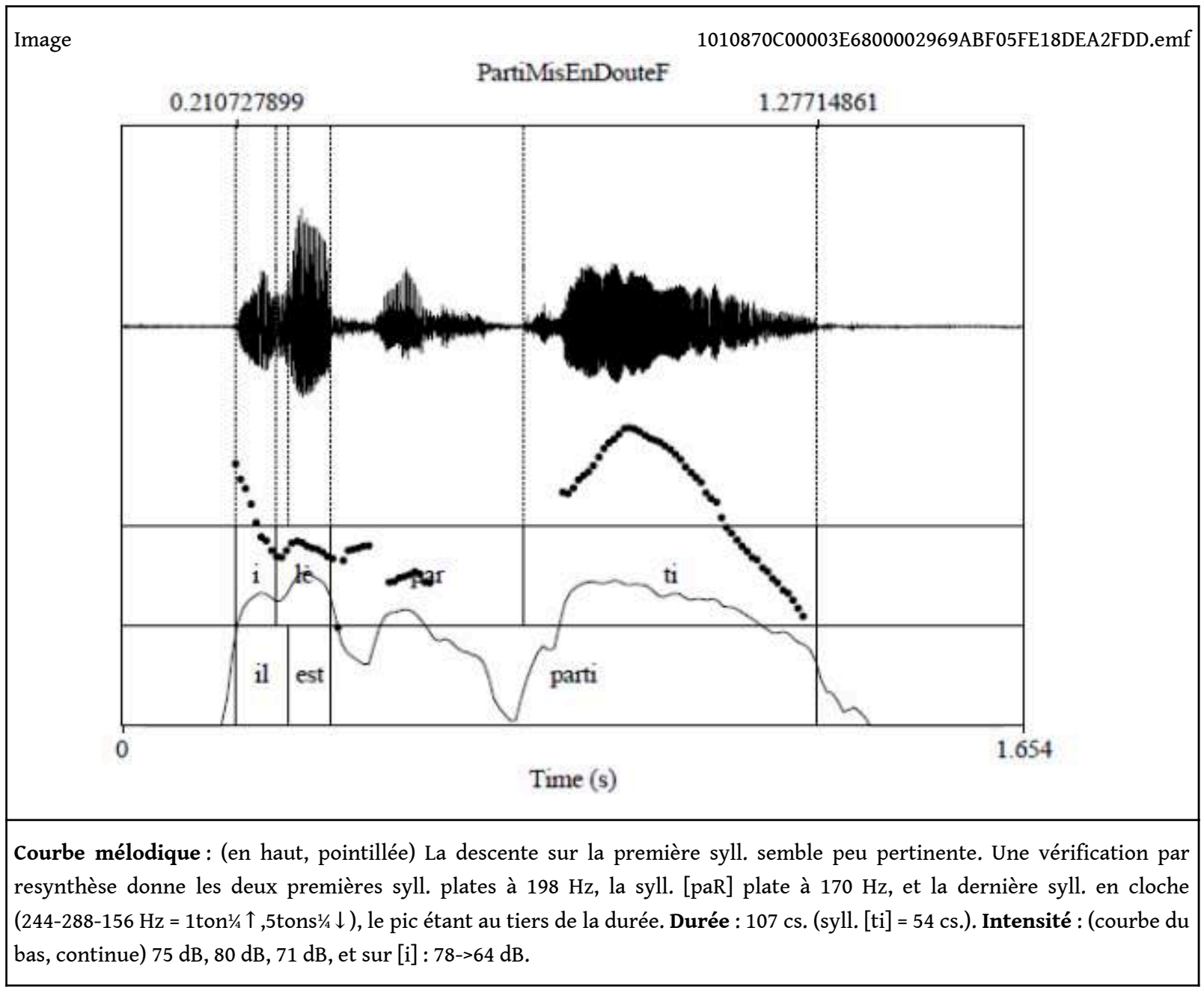

3 Cette intonation n'est pas étudiée, à notre connaissance. Ainsi, elle ne fait pas partie des intonations décrites oppositivement par P. Delattre (1966) ou Ph. Martin (2009), ni nettement de celles, nombreuses, répertoriées par M. Martins-Baltar (1977) à partir de la séquence il est parti, que nous avons reprise pour cette étude. Pour varier les données, nous avons également enregistré diverses séquences de différentes longueurs (de "Moi» à "Le chien reste dehors»), permettant également de varier les modalités, en intégrant par exemple l'injonction - non réalisable avec Il est parti. Ainsi, nous avons testé la MDM avec entre autres la séquence trisyllabique [SS3edœY $]^{4}$ (sans sourdes sur la syllabe tonique), dont le contexte construit est le suivant :

Léa - Hello Domi, ça va ? Ça se passe mieux depuis que tu as changé d'heure?

Domi - Changé d'heure...

Léa - Oui bon d'accord 15 minutes c'est pas énorme mais en attendant la restructuration tu peux débriefer Carole.

4 Soulignons que $\mathrm{P}$ peut se réduire à un seul mot ou syntagme, par exemple : Bien payé..., Costaud... 


\section{Nature de l'intonation MDM}

5 Cette intonation peut s'inscrire dans le paradigme des intonations à fonction modalisatrice. Les intonations à valeur modale considérées par Ph. Martin (2009: 89) comme fondamentales sont l'Assertion, la Question, l'Évidence, le Doute, l'Injonction et la Surprise. Mais ce modèle d'une élégante simplicité laisse de côté nombre de modalités dont la liste est largement ouverte : Demande de confirmation, Expectative, Désemparement, Étonnement sceptique, Incrédulité, Narguerie, Rejet...

6 Si l'on s'intéresse aux modalités en tant que marquage de l'attitude du locuteurénonciateur quant à une proposition $\mathrm{P}$, la limite $\mathrm{du}$ «métalinguistique » peut être interrogée.

7 Certaines modalités (Assertion, Question, Évidence, Désemparement...) renvoient nettement au monde et non à un (premier) discours tenu sur le monde.

8 D'autres sont clairement un commentaire sur un dit précédent. Outre l'intonation MDM, deux autres cas de figure nous paraissent être de nature métalinguistique :

9 - l'Écho parodique qui vient discréditer un dit précédent en le ridiculisant: Oh lui eh! « J'ai la priorité ", [je t'en ficherais, moi !] ;

10 - la reprise "Arrêt sur image » interrompant l'allocutaire à des fins de clarification : " C'est son droit... » [Attends, qu'est-ce que tu veux dire par là ?].

11 Mais certaines modalités peuvent opérer aussi bien sur $\mathrm{P}$ comme première mention que comme reprise. Il en va ainsi de ce que nous appelons "Étonnement dubitatif », qui peut survenir aussi bien au vu d'une situation (j'ouvre la fenêtre et ne vois pas la voiture de Paul) qu'en reprise d'un énoncé précédent, comme dans le contexte cidessous :

A. - Dis-donc, t'as pas vu Paul?

B. - Oh je crois qu'il est parti, je le vois plus depuis un moment.

A. - Il est parti ? C'est bizarre, je devais le ramener, moi, en principe.

Dans ce dernier cas, cette intonation peut s'interpréter comme « que me dis-tu là ? » Il en va de même pour l'Expectative qui, s'il s'agit d'une reprise, s'interprétera comme " pas si sûr, tu devrais te poser la question ». Un contexte possible :

A - On a gagné!

B - On a gagné... Peut-être, mais attendons l'affichage des scores, tu veux bien?

13 Mais ce qui est en jeu c'est « est-il ou non parti », «avons-nous ou non gagné », sans qu'il s'agisse d'une critique sur le fait que l'allocutaire a proféré P. On peut donc avoir un phénomène de reprise sans valeur métalinguistique, et le statut de reprise de $\mathrm{P}$ est une des conditions nécessaires pour que l'intonation puisse avoir une valeur métalinguistique, sans être une condition suffisante :

\begin{tabular}{|c|c|c|c|}
\hline & nécessaire & possible & exclu \\
\hline mécanisme de reprise & $\begin{array}{l}\text { MDM } \\
\text { Écho parodique } \\
\text { Arrêt sur image }\end{array}$ & $\begin{array}{l}\text { Étonnement dubitatif } \\
\text { Prise en considération } \\
\text { Stupéfaction incrédule } \\
\text { Expectative... }\end{array}$ & $\begin{array}{l}\text { Question } \\
\text { Demande de confirmation } \\
\text { Assertion } \\
\text { Évidence... }\end{array}$ \\
\hline
\end{tabular}


On pourrait être également tenté de réduire la spécificité de la MDM à un caractère suspensif, mais cela ne nous paraît pas crucial, dans la mesure où l'on pourrait attribuer ce même caractère à d'autres intonations ne relevant pas d'une valeur métalinguistique, comme :

- Prise en considération : Au commissariat de police :

- Un enquêteur : Monsieur l'inspecteur, un témoin nous affirme avoir vu le suspect sortir par la terrasse avant la fin du discours du fils de la victime.

- L'inspecteur: Ah ah. Il est parti... [ Eh bien, nous allons voir comment il expliquera qu'il

ait pu être encore là à $20 \mathrm{~h}$ !] Conduisez-le en salle d'interrogatoire.

- Demande de confirmation:

Il est parti ? [je ne me trompe pas ?]

- Délibération: Le locuteur, responsable des emplois du temps dans l'institut de formation, arrive à son bureau et lit une note informant que Mr Paul Durand a pris sa retraite anticipée et ne donnera pas cours cette année :

Il est parti... [voyons voyons... comment je vais arranger le planning ?] ressortissant à ce que décrit J. Authier-Revuz (2003: 92-93) comme «non-coïncidence entre les mots et les choses » (ces mots ne disent pas la réalité de l'état de choses dont nous parlons) et «non-coïncidence entre interlocuteurs » (pour moi P ne signifie pas ce que je reconstruis comme ce que $P$ signifie apparemment pour toi). Une intonation peut donc s'inscrire dans un fonctionnement proche de celui des marqueurs discursifs.

17 - Soulignons que cette valeur modale de mise en doute peut reposer uniquement sur l'intonation, bien qu'elle soit explicitable dans la suite du discours.

18 - On soulignera également que, des trois cas que nous avons mentionnés avec reprise nécessaire, seule la MDM autoriserait, à certaines conditions, l'auto-reprise - il faut alors opérer une rupture par un marqueur discursif :

Alors tu imagines bien qu'avec tout ça il est parti. Enfin, il est parti... je me comprends.

19 Mais dans la plupart des cas il s'agit d'une hétéro-reprise.

\section{Quelle description pour des intonèmes à valeur modale et/ou attitudinale?}

Peut-on défendre une approche opératoire ${ }^{6}$ de l'intonation à valeur modale ? C'est aller contre une tendance à laquelle même I. Fònagy n'échappe pas, qui est de conférer à l'intonation un caractère marginal ou périphérique, en tout cas non formalisable :

I think [...] that the essential function of intonation consists in conveying pre-verbal messages : attitudes and emotions, not covered by other linguistic means. We have to reckon, at the same time, with the no less basic endeavor of intonation to move towards higher levels of linguistic processing, even if it cannot reach the level of conceptual organization. (Fònagy 1989: 83-84)

21 Il convient tout d'abord de proposer une analyse sémantique des modalités permettant de dépasser le simple étiquetage qui n'explique ni ne déploie rien. Il en va ainsi de l'appellation "implication », qui nous intéresse particulièrement ici car on pourrait être tenté de l'appliquer à la MDM. C'est l'une des dix intonations de base de P. Delattre, 
reprise telle quelle ou comme composante par nombre d'auteurs ${ }^{7}$, bien que cette étiquette relativement floue ne contribue pas toujours à la rigueur de l'analyse.

Par exemple, la thèse de Cristel Portes intitulée «Prosodie et économie du discours : spécificité phonétique, écologie discursive et portée pragmatique de l'intonation d'implication » repart de la proposition de $\mathrm{P}$. Delattre ${ }^{8}$, en l'inscrivant dans la lignée des travaux de l'Ecole de Genève. «Delattre justifie son choix de la dénomination intonation d'implication en affirmant que l'usage de ce contour établit un sous-entendu que l'auditeur doit décrypter. L'intonation d'implication commande chez l'auditeur l'effectuation de ce que Roulet va appeler un parcours inférentiel. $»^{9}$ Or, tout énoncé génère dans la reconstruction de son sens des opérations diverses, dont quantité d'inférences de toute nature. A notre sens, l'intonation MDM ne «sous-entend » rien, elle marque explicitement une mise en cause de l'adéquation de $\mathrm{P}$ à rendre compte de l'état de choses dont on parle. Il en va de même pour toutes les autres modalités que nous avons étudiées, le recours à un quelconque «sous-entendu » ne nous paraissant pas nécessaire.

Les travaux de C. Portes s'inscrivent dans un important courant d'étude de l'intonation ${ }^{10}$, et s'appuient sur l'observation de corpus conversationnels. L'auteure a fait repérer par des experts des occurrences de ce qui est appréhendé comme cette même intonation "implication" dans un corpus de débats médiatiques. Il se dégage de l'étude qu'au moins deux formes différentes de courbes doivent être distinguées, variant quant à la position du pic de hauteur le long de la courbe en cloche. D'autre part au fil du texte on voit que des valeurs d'évidence, d'ironie, de doute, d'emphase convaincue, de demande d'assentiment peuvent toutes ressortir à l'une de ces courbes : la notion d'implication est donc insuffisamment discriminante. Il ne serait d'ailleurs pas surprenant que des MDM fassent partie du corpus analysé, l'auteure soulignant le contexte polémique d'apparition de l'intonation d'«implication ", et observant: "Formulée, désignée ou implicitée, on voit bien que la parole d'autrui représentée par celle du locuteur prend une importance particulière dans ces épisodes polémiques .» (Portes 2008: 152)

Le repérage de schémas intonatifs dans des corpus spontanés est une recherche cruciale, mais doit, à notre sens, être précédé par une mise au jour d'intonèmes par production contrôlée, validation par reconnaissance auditive, et vérification par synthèse. Au stade où en sont nos travaux, nous pouvons clairement opposer la MDM à d'autres intonations présentant un contour "en cloche », et formuler des hypothèses quant aux correspondances entre différences sémantiques et différences formelles. Pour ce faire, le passage par une approche non différenciée en termes d' "implication » ne nous paraît pas indispensable.

Pour arriver à des catégories plus fines et plus à même de différencier les intonations, nous proposons de prendre en compte différentes caractéristiques sémantiques de telle ou telle modalité ou attitude ${ }^{11}$ exprimée par l'intonation, par exemple :

- La relation prédicative en jeu (soit P) est-elle ou non en rapport avec autre que $\mathrm{P}$ ou non $\mathrm{P}$ (désormais $\mathrm{P}^{\prime 12}$ ), et si oui de quelle façon?

- A-t-on, sur l'une ou l'autre valeur (P ou P'), stabilité, instabilité ou stabilisation?

- Quelle est la source de la construction de P': la situation, l'allocutaire, le locuteur?

- Quelles sont les instances subjectives en jeu dans la prise en charge de P, de P' ou de l'opposition $\mathrm{P} / \mathrm{P}$ '? 
$(2008: 52-53)$

orientation descendante
niveaux bas
attitude monologale
centrage sur le point de vue
personnel de l'énonciateur
rupture avec la consensualité
préalable
consensualité, accord, émotion
évacuée

orientation descendante

orientation montante

niveaux hauts

attitude dialogale

convergence des points de vue

discours adressé à autrui de manière

consensuelle

confrontation à soi et aux autres

G. Caelen-Haumont

niveau 4 [aigu] : lieu de la remise en cause, lié à l'émotionnel

Pour parvenir à identifier des formes intonatives opposables, nous avons procédé par une comparaison systématique d'une quinzaine de courbes dans des conditions de paires minimales ${ }^{14}$ permettant de neutraliser les interférences microprosodiques et d'isoler la valeur modale. Cette analyse oppositive de diverses modalités, qui s'inscrit dans la lignée des travaux de P. Delattre (1966) et M. Martins-Baltar (1977), nous a permis de formuler des hypothèses quant aux correspondances entre formes de l'expression et caractéristiques sémantiques. Notre propos n'est pas dans ce texte de les développer, mais de les mettre à l'épreuve dans l'analyse de la MDM.

\section{Spécificité de la MDM}

\subsection{Un patron intonatif particulier}

L'intonation Mise en doute métalinguistique présente une courbe en cloche, qui se distingue nettement de la courbe d'Évidence, comme on peut voir dans les deux courbes ci-dessous correspondant l'une et l'autre à la séquence [SSFedZY] : pour la MDM la montée ne s'opère que sur la dernière syllabe [dZY], alors qu'elle s'amorce sur la pénultième $[\mathrm{Fe}]$ pour l'Évidence ${ }^{15}$ :

Mise en doute métalinguistique : 


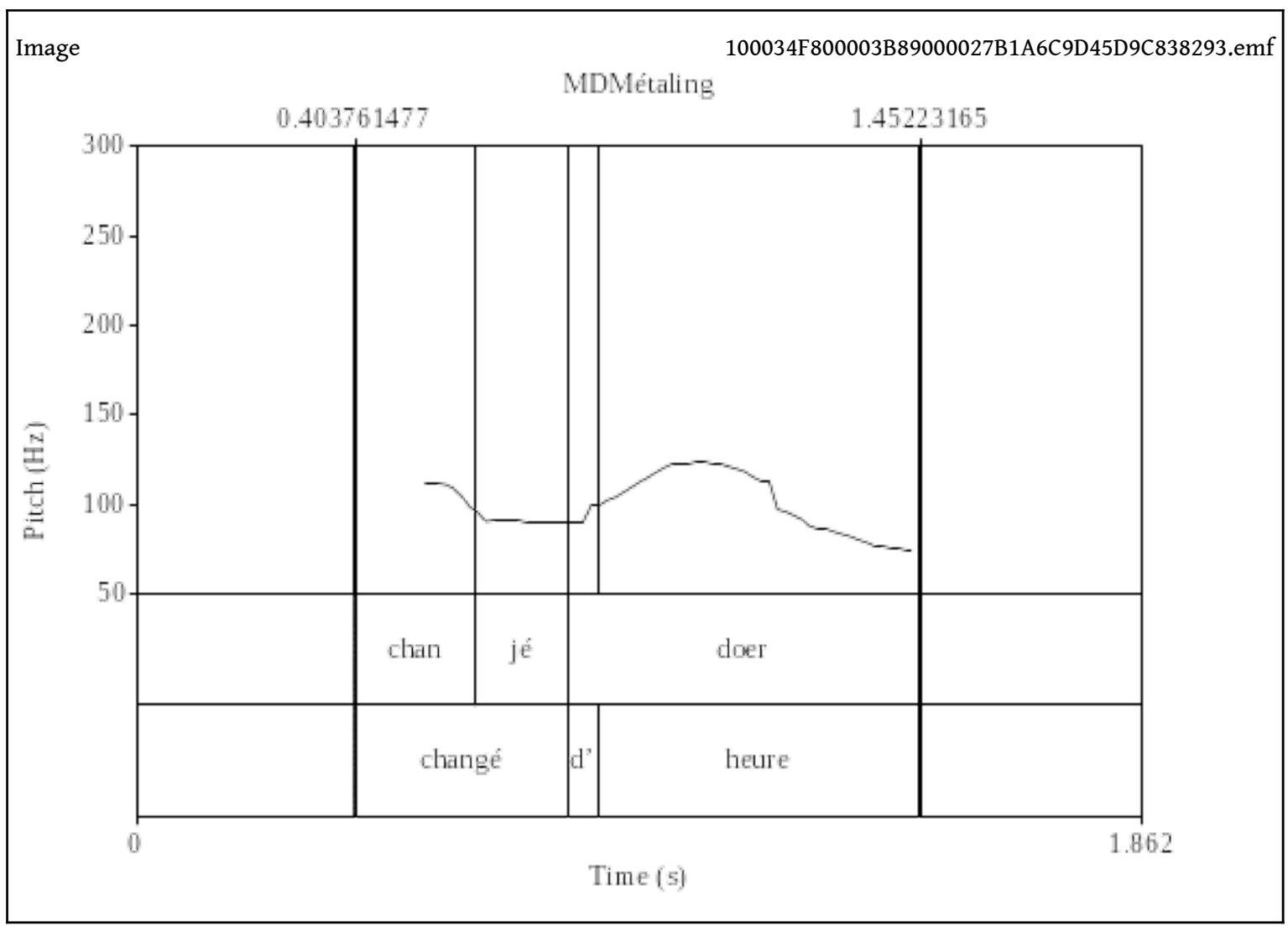

Contexte : - Anna : Hello Domi, ça va? Ça se passe mieux depuis que tu as changé d'heure?

- Domi : Changé d'heure...

- Anna : Quoi?

- Domi : J'avais demandé une autre tranche pas un petit décalage.

- Anna: Oui bon d'accord 15 minutes c'est pas énorme mais en attendant la restructuration tu peux au moins débriefer Carole.

Évidence ${ }^{16}$ : 


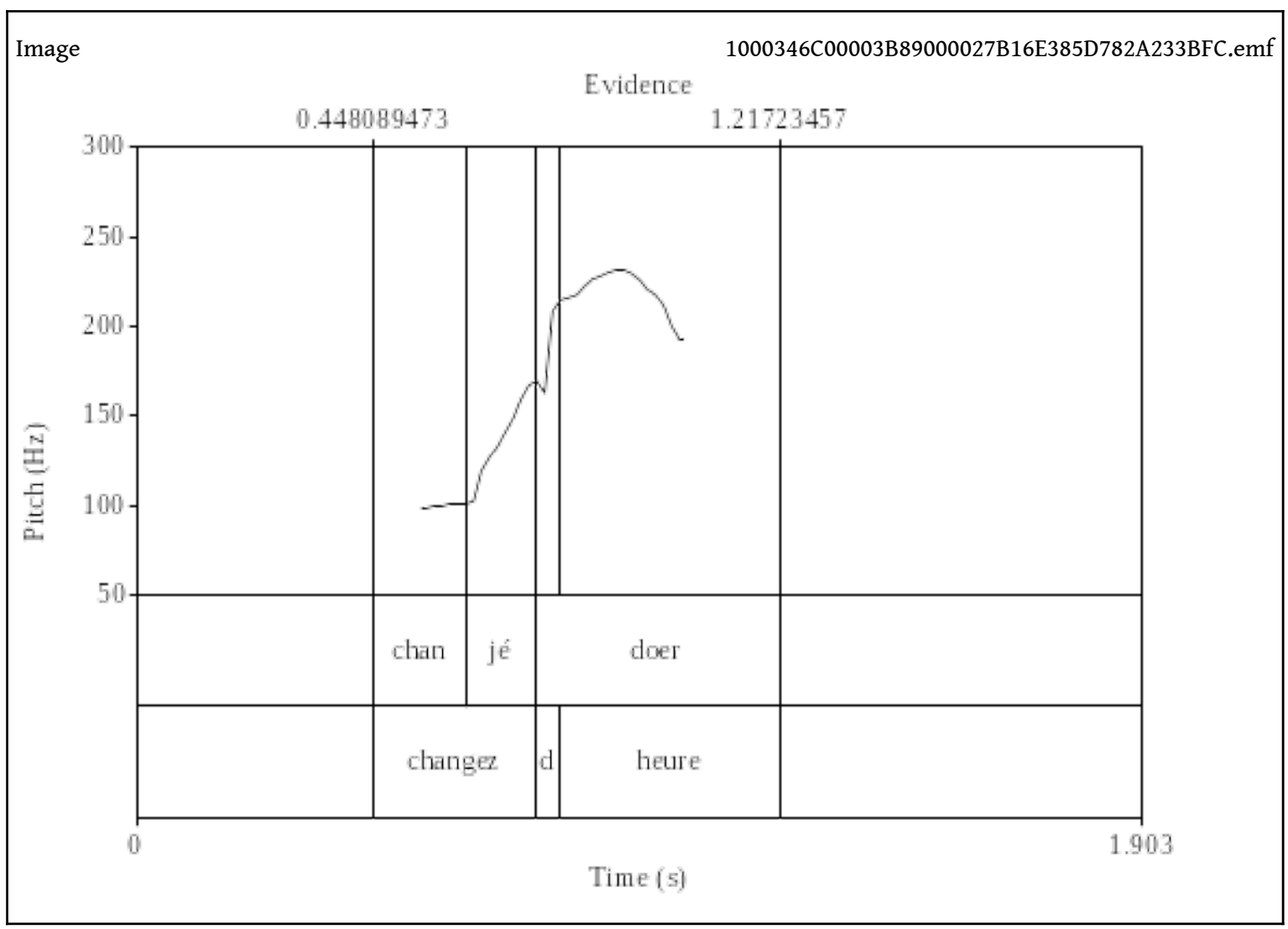

Contexte : - Domi : Salut Claude, réunion urgente demain en fin d'après-midi !

- Claude : Ah mince... Tu sais ces rendez-vous chez ton psy qui nous font vraiment du bien à Léa et moi. Déjà que Léa a sa consultation demain matin qui saute à cause de son exam, et moi cette réunion ça tombe en même temps que la mienne!

- Domi : Ben c'est pas compliqué voyons. Changez d'heure ... ! Pour une fois, il sera sûrement d'accord.

31 Ceci plaide pour la prise en considération du groupe prosodique dans son ensemble et pas seulement de la syllabe tonique. Toutefois la hauteur de la syllabe pénultième n'a pas de signification en soi. Elle permet d'ajuster deux éléments qui pourraient être contradictoires. Ainsi, si l'on doit avoir une montée relativement marquée, tout en n'atteignant pas le registre aigu ou suraigu, une baisse sur la pénultième permet la combinaison de ces deux traits ${ }^{17}$.

Comparons cette dernière avec la MDM, le contexte proposé pour cette valeur d'Expectative étant :

A - On a essayé de faire les groupes mais Marie s'est inscrite et du coup je pense que Paul est parti. À mon avis ça va pas fonctionner.

B - Il est parti... Ça reste à voir. Attendons les inscriptions demain. 


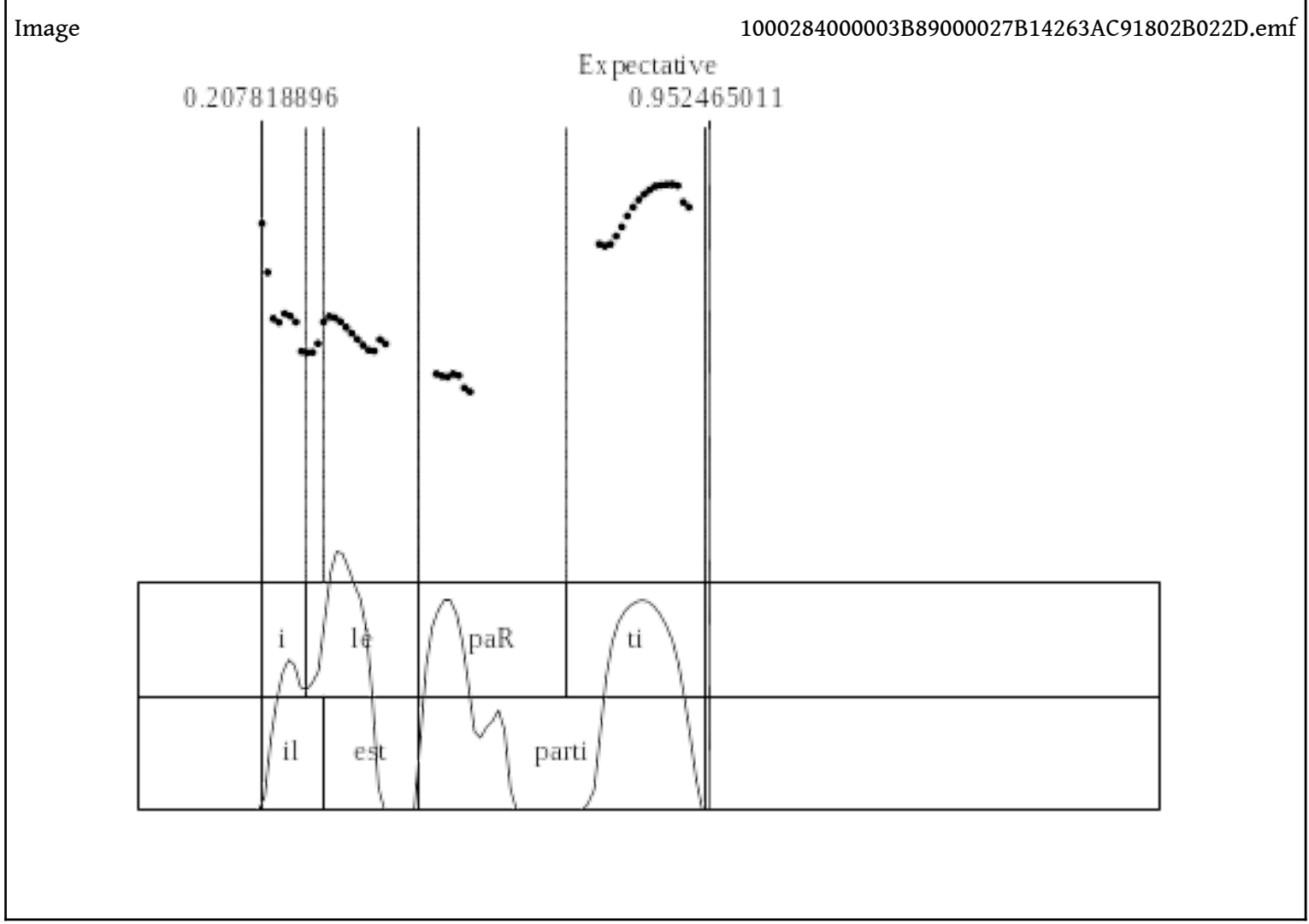

Rappel de la courbe $\mathrm{MDM}^{18}$ :

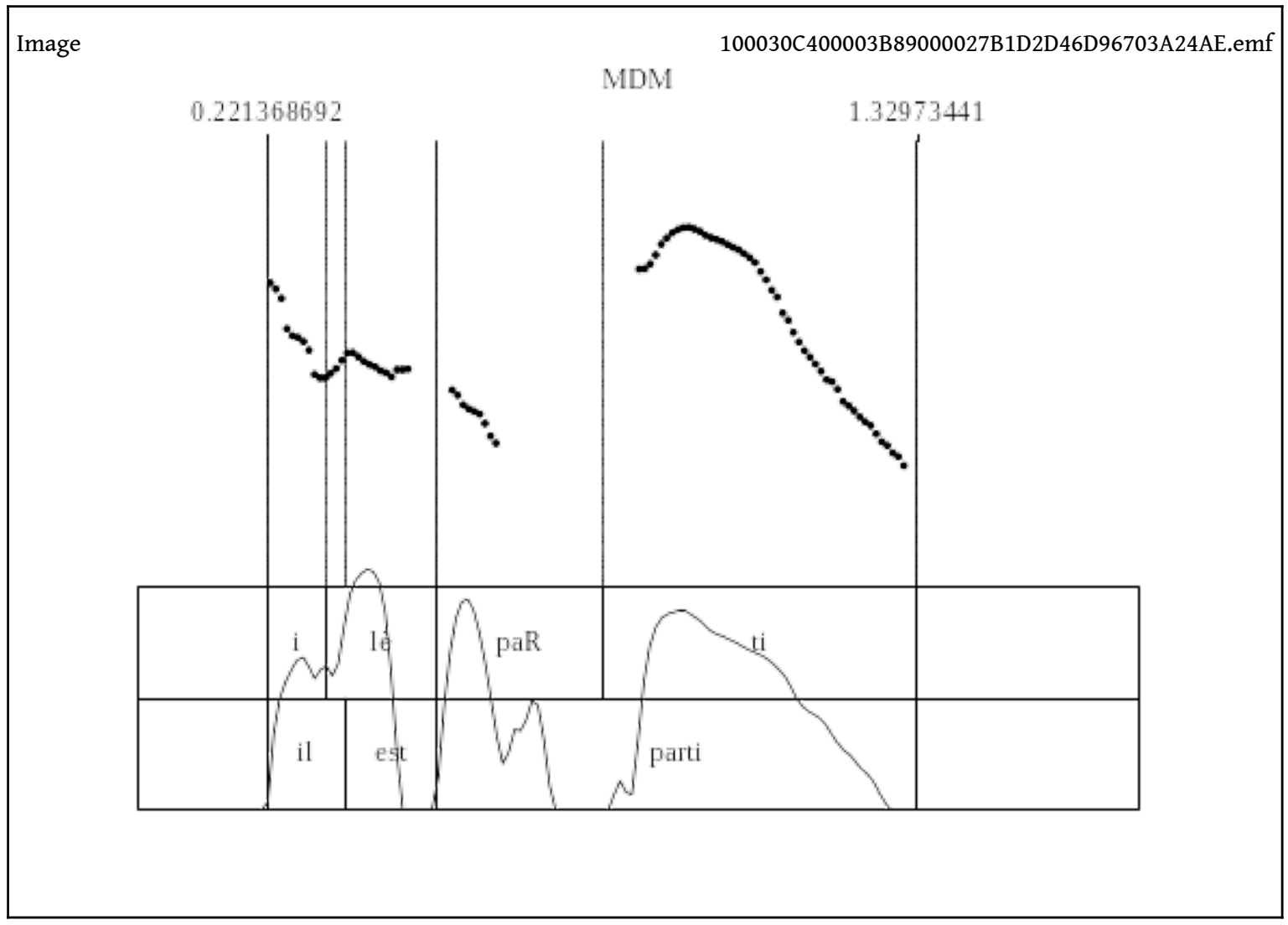



rattaché à un préfixe :

«[...] les préfixes n'ont pas de marques dans le texte indiquant la modalité de l'énoncé comme le noyau ou les suffixes. Pour garder leur caractère de préfixe, la structure prosodique qui y est associée ne peut pas non plus indiquer de modalité, qui reste l'apanage du noyau, même effacé. Il en résulte que le contour final d'un préfixe "suivi » d'un noyau effacé ne peut pas non plus indiquer de modalité, et devra contraster avec les contours montants de continuation et aussi avec les contours terminaux. Il ne sera donc ni descendant comme le contour terminal de modalité déclarative, ni montant comme celui de modalité interrogative, et devra également se différencier de toutes les variantes de contours de modalité et aussi des contours qui terminent normalement les préfixes dits de "continuation majeure ». Il sera donc similaire aux contours de fin de préfixe, et éventuellement pourvu d'un mouvement en cloche en fin de montée pour indiquer un renvoi, une allusion au contexte. » (Martin 2009: 178)

On aurait donc une courbe non significative sémantiquement, uniquement motivée par la règle d'inversion de pente (Martin 1981: 265), qui pose qu'un segment "préfixe» dans la dépendance d'un segment «noyau » se termine par une pente inverse de celle qui termine le segment noyau. Si le constituant élidé se termine potentiellement par une descente, alors la syllabe accentuée du préfixe fait l'objet d'un mouvement de montée, et inversement.

Cela nous paraît infirmé par la diversité des contours qui pourraient être considérés comme ressortissant à un tel mécanisme. Par exemple Expectative, Étonnement dubitatif, Reprise ironique, Arrêt sur image, Prise en considération ou ce que nous appelons à la suite de M. Martins-Baltar "Délibération ${ }^{22}$ ont chacun un contour différent.

Corela, HS-31 | 2020 

l'allongement caractéristiques de la courbe de MDM, comme dans ces exemples repérables chez Ph. Martin lui-même :

- et le pire c'est de pas pouvoir la retoucher quoi ça me tue ça me tue mais bon si tu me dis que tu as l'ordinateur

- et le pire c'est d'être encore toute seule pour tout faire ça ça me tue mais bon si Paul arrive demain (Martin 2009: 178)

En effet, la courbe de la MDM redescend au niveau grave de la finalité et ne correspond donc pas au «mouvement en cloche en fin de montée » décrit pour les ellipses par Ph. Martin.

Notons au passage que, ayant observé que la "possibilité pour l'intonation d'implication de précéder un segment d'énoncé désaccentué s'avér[ait] l'un des traits les plus souvent mis en avant par les analyses [de divers auteurs] », C. Portes (2008: 29) convient qu'elle "ne pourr[a] rien dire de ce fait en raison de l'absence totale d'exemples de ce type dans [son] corpus. »

\subsection{Forme de la MDM et opérations énonciatives}

On peut décrire le contour de la MDM comme présentant les caractéristiques suivantes : forme en cloche, registre global médium, saut entre la pénultième et la tonique, allongement marqué de la tonique. La forme en cloche est de type (ascendante)-descendante ${ }^{23}$ : par cette notation nous voulons signifier que le pic de hauteur étant au premier tiers de la syllabe, la durée nettement supérieure de la descente donne une impression globale de courbe descendante.

Il n'est pas question d'exposer ici un modèle complet (tel qu'il se présente dans l'état actuel de notre réflexion). Nous mentionnerons seulement les correspondances suivantes (que nous donnons à titre d'hypothèses), pertinentes pour l'intonation qui nous intéresse ${ }^{24}$ :

- Un mouvement ascendant marquerait une instabilité : la relation prédicative $\mathrm{P}$ est mise en balance avec P'. La question totale (non rhétorique, non vérificatoire) est caractéristique de cette instabilité, maximale car alors aucune des deux valeurs n'est privilégiée.

- Un mouvement descendant jusqu'au grave irait de pair avec une stabilité de P. L'assertion simple en est caractéristique, $\mathrm{P}$ étant posée sans que P' ait un mode de présence.

- La combinaison des deux mouvements (par exemple une forme en cloche) peut produire, selon les cas, tel ou tel rapport entre instabilité (P, P') et stabilité (sur P ou sur P').

- Un écart de hauteur en rupture marquerait une discordance entre deux points de vue. La stupéfaction incrédule en est caractéristique, avec l'alternance syllabe basse - syllabe haute sur tout l'énoncé.

- Un registre global médium ( $v s$ aigu) marquerait que le point de vue sur les valeurs en concurrence est de l'ordre de l'évaluation intellectuelle, du calcul et non de la réaction irréfléchie.

- Une durée remarquable de la syllabe tonique marquerait que le locuteurénonciateur est seul constructeur d'une opposition P / P'.

Une relation prédicative $\mathrm{P}<\mathrm{i}$ est parti> peut être envisagée comme un domaine notionnel tel que défini par Antoine Culioli, structuré en différentes zones : Intérieur $(\mathrm{P})$, Extérieur $\left(\mathrm{P}^{\prime}\right)$, Frontière (ni vraiment $\mathrm{P}$ ni vraiment $\left.\mathrm{P}^{\prime}\right)$, avec une position décrochée où les deux sont envisageables (notée IE, soit $\mathrm{P}, \mathrm{P}^{\prime}$ ). L'intonation peut, comme d'autres 
formes linguistiques (verbes modaux...), marquer l'hétérogénéité des positions sur le domaine de P. Ainsi la surprise, fortement ascendante, va mettre en saillance le fait que sur le plan $\mathrm{T}$ (donnée spatio-temporelle ou traitée comme telle), on a $\mathrm{P}$, et sur le plan $\mathrm{S}$ (repérage subjectif), c'est P' qui est attendu. Les deux zones I et $\mathrm{E}$ sont donc pointées, ainsi que leur discordance.

En rapport avec les hypothèses posées plus haut, il nous paraît possible de décrire l'intonation de Mise en doute métalinguistique de la façon suivante :

- Registre global médium : recul intellectuel.

- Forme en cloche : mise en saillance du zonage du domaine de $\mathrm{P}^{25}$.

- Cloche (ascendante)-descendante : rabattement sur une valeur stabilisée, celle privilégiée par le locuteur-énonciateur. La redescente marquée jusqu'au grave construit/traduit une stabilisation sur la valeur prise en charge par le locuteur - en l'occurrence, P' (à l'inverse de l'évidence où c'est sur P que s'opère la stabilisation).

- Saut entre [paR] et [ti] : discordance (caractéristique partagée avec l'Étonnement dubitatif, et contrairement à l'Évidence ou à la Demande de confirmation où le locuteur «tient pour $\mathrm{P}$ »). L'écart mélodique entre la pénultième et la finale met ici en saillance la singularité du point de vue du locuteur (par opposition avec un autre point de vue). C'est une différence importante avec l'Évidence, où le point de vue du locuteur validant P (au regard d'une instabilité potentielle (P,P') posée par (ou attribuée à) l'allocutaire) est donné comme partagé par tout sujet raisonnable.

- Allongement marqué de la durée de la tonique : le locuteur-énonciateur est seul constructeur de la mise en balance de $\mathrm{P}$ avec $\mathrm{P}$.

On résumera les considérations précédentes dans le tableau suivant, que nous proposons sous réserve de recherches plus développées :

\begin{tabular}{|c|c|c|c|}
\hline Plan formel & Plan sémantique & Partagé avec & Opposé à \\
\hline courbe en cloche & zonage du domaine de $\mathrm{P}$ & $\begin{array}{l}\text { Évidence } \\
\text { Demande de } \\
\text { confirmation } \\
\text { Étonnement } \\
\text { dubitatif }\end{array}$ & $\begin{array}{l}\text { Assertion } \\
\text { Question }\end{array}$ \\
\hline $\begin{array}{l}\text { courbe de type } \\
\text { (ascendante)-descendante }\end{array}$ & $\begin{array}{l}\text { rabattement sur une valeur } \\
\text { stabilisée }\end{array}$ & Évidence & $\begin{array}{l}\text { Étonnement } \\
\text { dubitatif } \\
\text { Expectative }\end{array}$ \\
\hline registre global médium & $\begin{array}{l}\text { recul } \quad \text { évaluateur } \\
\text { gestionnaire du locuteur }\end{array}$ & $\begin{array}{l}\text { Évidence } \\
\text { Expectative }\end{array}$ & $\begin{array}{l}\text { Stupéfaction } \\
\text { incrédule }\end{array}$ \\
\hline $\begin{array}{l}\text { saut entre pénultième et } \\
\text { tonique }\end{array}$ & discordance marquée & $\begin{array}{l}\text { Étonnement } \\
\text { dubitatif } \\
\text { Stupéfaction } \\
\text { incrédule }\end{array}$ & $\begin{array}{l}\text { Évidence } \\
\text { Délibération }\end{array}$ \\
\hline $\begin{array}{l}\text { allongement marqué de la } \\
\text { tonique }\end{array}$ & $\begin{array}{l}\text { locuteur-énonciateur } \\
\text { constructeur de } \mathrm{P} / \mathrm{P}^{\prime}\end{array}$ & $\begin{array}{l}\text { Évidence } \\
\text { Délibération }\end{array}$ & Expectative \\
\hline
\end{tabular}


Il y a beaucoup de formes de courbes qui peuvent être dites "en cloche", et une description fine s'impose. Ainsi le contour de la MDM est-il nettement différent de celui de l'Évidence, de la Prise en considération, de l'Expectative. On ne saurait non plus le confondre avec le mélisme «emphatique-convivial» dans l'aigu ${ }^{26}$, une des caractéristiques prégnantes de la MDM étant justement le recul qu'elle mobilise, en tant qu'acte de nature purement métalinguistique.

51 Ceci n'empêche pas une forte variation mélodique, que l'on ne saurait donc toujours associer à "l'émotion », celle-ci devant être nettement dissociée du marquage de la subjectivité, c'est-à-dire de la construction par des opérations énonciatives de l'engagement du locuteur-énonciateur comme source singulière d'un point de vue en relation d'altérité potentielle avec un autre point de vue.

\section{Remarques conclusives}

52 Nous espérons que les observations précédentes arguent en faveur de la non séparation des domaines (syntaxe, sémantique lexicale, analyse prosodique) dans l'analyse de la construction du sens au sein des énoncés.

Parallèlement aux fonctions démarcative et distinctive ${ }^{27}$ qui lui sont spécifiques, la prosodie partage avec les unités de première articulation toutes les autres fonctions. Nous avons vu que le caractère métalinguistique ou non de certaines modalités peut être indécidable, tout comme cela se produit pour certaines tournures ou expressions (par exemple y a-t-il ou non un aspect métalinguistique dans tous les emplois de à vrai dire, ou dans la construction son général de mari ?). Il fallait donc s'attendre à ce que, de même que pour certains marqueurs discursifs (e.g. si on peut dire), la prosodie puisse à elle seule exprimer un commentaire purement métalinguistique. C'est le cas pour l'intonation que nous avons présentée ici, qui permet de gloser un énoncé précédent à travers la seule reprise de cet énoncé.

54 Si certaines opérations qu'on pourrait dire métaénonciatives peuvent s'effectuer hors conscience dans l'énoncé, et ressortissent à ce qu'Antoine Culioli associe par endroits à «l'épilinguistique ${ }^{28}$ ", le point de vue métadiscursif sous-jacent à l'intonation MDM est nécessairement conscient. Cette intonation traduit une attitude de recul intellectuel et de mise en perspective d'un dire autre (même si c'est le sien propre, que l'on réenvisage alors avec distance).

Signalons pour finir que, contrairement aux autres modalités que nous avons étudiées, la MDM est difficilement identifiée et réalisée par certains locuteurs - nous avons pu le constater auprès de nos étudiants. Mais après une période de familiarisation, elle peut être maîtrisée en reconnaissance comme en production.

Une enquête sur la variation diastratique des emplois spontanés de cette forme de commentaire métalinguistique purement intonatif serait bienvenue, mais un protocole de collecte des données intégrant des variables socio-culturelles parait très difficile à mettre en place. 


\section{BIBLIOGRAPHIE}

Authier-Revuz J. (2003). Le fait autonymique : Langage, langue, discours, quelques repères. In Authier-Revuz J. et al. (eds) Parler des mots, le fait autonymique en discours. Presses Sorbonne Nouvelle. 67-96.

Caelen-Haumont G. (2002). Prosodie et dialogue spontané : valeurs et fonctions perlocutoires du mélisme. Travaux Interdisciplinaires du Laboratoire Parole et Langage d'Aix-en-Provence 21. 13-24.

Caelen-Haumont G. (2008). Prosodie et sens. Une approche expérimentale Vol. 2. Paris : L'Harmattan.

Culioli A. (1990). Pour une linguistique de l'énonciation T. 1. Paris : Ophrys.

Delattre P. (1966). Les Dix intonations de base du français. The French Review 40/1. 1-14.

Fónagy I. (1989). On status and functions of intonation. Acta Linguistica Hungaria 39. 53-92.

Fónagy I. et Bérard E. (1973). Questions totales simples et implicatives en français parisien. Studia Phonetica 8. Interrogation et intonation. 53-97.

Léon P. (1970). Systématique des fonctions expressives de l'intonation. Studia Phonetica 3. 57-74.

Martin P. (1981). Pour une théorie de l'intonation. In Rossi M., Di Cristo A., Hirst D., Martin Ph. \& Nishinuma Y. (eds) L'Intonation, de l'acoustique à la sémantique. Paris : Klincksieck. 234-271.

Martin P. (2009). Intonation du français. Paris : A. Colin

Martins-Baltar M. (1977). De l'énoncé à l'énonciation : une approche des fonctions intonatives. Paris : CREDIF.

Mertens P. (1987). L'Intonation du français. De la description linguistique à la reconnaissance automatique. Thèse. Katholieke Universiteit Leuven.

Morel M.-A. et Danon-Boileau L. (1998). Grammaire de l'intonation. L'exemple du français. Paris : Ophrys.

Normand C. (2012). La notion d'ajustement dans le métalangage d'Antoine Culioli. In FilippiDeswelle C. (éd.) L'Ajustement dans la Théorie des Opérations Énonciatives d'Antoine Culioli. Épilogos 3. ERIAC. Université de Rouen. 29-38.

Portes C. (2004). Prosodie et économie du discours : Spécificité phonétique, écologie discursive et portée pragmatique de l'intonation d'implication. Linguistique. Université de Provence -Aix-Marseille I.

Français. Dans HAL : <tel-00296742v2>

Rouskov-Low J. (1991). Prosodie et domaine notionnel : quelques remarques. Cahiers Charles V 13. Travaux de linguistique énonciative. 149-162.

Rouskov-Low J. (1993). Iconicité énonciative des marqueurs prosodiques. Faits de langue 1. Motivation et iconicité. 215-222.

\section{NOTES}

1. Nous n'employons pas «métalinguistique » dans l'acception culiolienne de «niveau auquel s'élabore un système formel de représentation des opérations à l'œuvre dans la construction du sens des énoncés » (Culioli, 1990: 155), mais plus simplement comme «caractéristique d'un dire commentant le dit en tant que dit ». 
2. Les locuteurs enregistrés, tout comme les auditeurs devant identifier les intonations, se basent sur un contexte non ambigu (que nous construisons) pour la production et la reconnaissance des intonations. Nous ne leur proposons aucune «étiquette » (voir exemples note suivante) supposée correspondre à tel ou tel de ces contextes, considérant inutile le recours à un métalangage qui, s'il est nécessaire pour la commodité de la désignation, n'a aucun caractère scientifique.

3. Cette réalisation (locutrice, environ 50 ans) a été validée par écoute et identification en concurrence avec huit autres intonations (Assertion, Assertion péremptoire, Question, Étonnement dubitatif, Surprise, Stupéfaction incrédule, Évidence, Délibération). Les autres intonations sur lesquelles nous avons travaillé sont pour l'instant : Désemparement, Demande de confirmation, Prise en considération, Prise en considération rusée, Narguerie, Écho parodique, Reprise ironique, Injonction, Arrêt sur image, Expectative. Ces dénominations n'ont pas de valeur autre que pratique et ne visent qu'à cerner au plus près la modalité en jeu. Nos courbes sont éditées sous le logiciel Praat.

4. La courbe figure plus loin.

5. Les séquences entre [] peuvent très bien ne pas être prononcées.

6. Articulant des caractéristiques formelles du signifiant à des opérations sémantiques.

7. Par exemple, «question implicative» (vs neutre) chez Fònagy \& Bérard (1973: 84) ; "patron montant-descendant » comme « marque intonative à contenu général implicatif » et « continuité et interrogative tonale implicatives » chez Léon (1970:59 et 68).

8. Le travail pionnier de Pierre Delattre est incontournable, et nous adhérons à sa démarche consistant à dégager des oppositions entre les contours en termes de traits. Mais son modèle est contestable à divers égards: mélange des fonctions démarcative et modalisatrice, variantes combinatoires (les deux " parenthèses » haute et basse), étiquetage des modalités peu explicite (« exclamation» et «implication»), pertinence contestable de l'opposition concave / convexe. Pour d'autres critiques, voir Mertens (1987: 154-165).

9. Portes (2004: 28). Les italiques sont de l'auteure.

10. Laboratoire Parole et Langage, Aix-en-Provence, UMR 6057.

11. Nous distinguons attitude et émotion. L'attitude renvoie pour nous à une prise de position du locuteur-énonciateur quant aux éléments en jeu dans le discours (la prédication, le monde à dire, les mots, l'altérité intersubjective) et peut éventuellement se conjuguer avec telle ou telle émotion (on peut exprimer la Surprise ou la Demande de confirmation avec colère, joie, peur...). En ce sens l'attitude est fondamentalement liée à l'activité langagière, ce qui n'est pas le cas de l'émotion.

12. Nous empruntons cette notation, qui permet de ne pas trancher a priori entre non $\mathrm{P}$ et autre que $\mathrm{P}$, à Antoine Culioli.

13. Chaque auteur ayant pour cadre un modèle qui a sa propre cohérence, non centré spécialement sur la modalité, il n'est nullement question ici de critiquer leurs travaux d'importance dans le champ de la prosodie. Nous voulons seulement souligner qu'il est difficile de se baser sur des expressions comme "ouverture ", " repli », " consensus " auxquelles n'est conféré aucun statut formel pour construire un raisonnement (opposant systématiquement les contours de différentes intonations modalisatrices) qui soit falsifiable.

14. À niveau segmental constant, ne variant que par l'intonation. Nous choisissons des énoncés compatibles avec une dizaine de modalités ou attitudes, par exemple C'est ça; c'est pour Cathy; votre valise...

15. Le rapport de la pénultième à l'antépénultième est inverse. Évidence : "chan » $=100 \mathrm{~Hz}$, " gez » = $150 \mathrm{~Hz}$ (centre de la voyelle), soit 3,5 tons plus haut. MDM : «chan » = $111 \mathrm{~Hz}$, « gé » = $89,7 \mathrm{~Hz}$, soit 1,5 ton plus bas. Locuteur du test (validé ensuite par identification auditive) : homme d'environ 50 ans.

16. Nous n'avons pas la fin de la courbe descendante car le [R] final est dévoisé. 
17. On observe également ce mécanisme pour les intonations Prise en considération et Expectative. Inversement, une montée brusque sur la pénultième permet, dans l'intonation Étonnement dubitatif, de réaliser une rupture avec la tonique qui repart du bas pour monter tout en restant dans un registre médium-grave.

18. Autre réalisation, d'un autre locuteur, que celle présentée au début, intonée dans les mêmes conditions que l'Expectative.

19. $\mathrm{MDM}$ : « est » : $189 \mathrm{~Hz}, 68 \mathrm{~dB}-$ « par » = 176-151 Hz, $65 \mathrm{~dB}, 28 \mathrm{cs}$. Expectative : « est » = $202 \mathrm{~Hz}$, $69 \mathrm{~dB}-$ « par » = 180-171 Hz, $65 \mathrm{~dB}, 26 \mathrm{cs}$. La hauteur de « il » est problématique, la voyelle n'étant pas nettement définie.

20. Point le plus haut : $256 \mathrm{~Hz}$ contre $272 \mathrm{~Hz}$, soit $\frac{1}{2}$ ton plus bas ; point le plus bas : $140 \mathrm{~Hz}$ contre $243 \mathrm{~Hz}$, soit 4,5 tons plus bas.

21. Il a dit qu'il était en train de se séparer, et ça fait un moment que je le vois plus avec Josiane. Après, est-ce qu'il est parti ... Il est peut-être planqué quelque part.

22. Nous ne pouvons ici présenter toutes les courbes et contextes correspondants. Pour certaines de ces modalités, on trouvera chez M. Martins-Baltar des contours approchants : Reprise ironique («assertion ironique » chez M-B. p. 61), Étonnement dubitatif (« doute » chez M-B. p. 59), Prise en considération (« considération » chez M-B. p 51), Délibération (p. 50).

23. Dans une courbe en cloche, diverses combinaisons des deux mouvements vont être possibles, générant des configurations où varieront la valeur privilégiée ( $\mathrm{P}$ ou P'), par le locuteurénonciateur ou par un énonciateur "de sens commun", l'écart plus ou moins grand entre l'attendu ou l'avéré... tous cas de figure que nous ne pouvons commenter ici.

24. Nous ne pouvons, dans le cadre de cet article, faire état de toutes les données et analyses qui nous permettent d'avancer ces correspondances. La proposition complète intitulée "Pour une description systématique et compositionnelle des intonations à valeur modale du français » est en cours de rédaction.

25. Ceci n'est pas sans évoquer la correspondance qu'établit J. Rouskov-Low (1991 et 1993) entre zonage du domaine notionnel (en référence aux travaux d'Antoine Culioli, voir en particulier 1990 : The Concept of Notional Domain, spécialement 70-71) et mouvements intonatifs. L'auteure rend compte entre autres des mouvements Fall, Rise et Fall-Rise de la langue anglaise dans les constructions positives, négatives ou interrogatives en termes de choix ou absence de choix entre les zones I ou E pointées sur le domaine notionnel de P. Nous ne reprenons pas les propositions de l'auteure, qui analyse le mouvement Fall-Rise comme une succession de choix d'un énonciateur qui nous paraît proche du locuteur - en tout cas dans la formulation, alors que nous préférons parler d'opérations abstraites dans un rapport non temporalisé. « FALL-RISE : dans les énoncés affirmatifs, l'énonciateur choisit d'abord l'intérieur du domaine notionnel (I), ensuite l'extérieur (I>E) et revient finalement à la frontière entre l'intérieur et l'extérieur (f). » (RouskowLow 1993: 220)

26. «Le mélisme, dans la mesure où il utilise le registre aigu, voire suraigu, est l'espace privilégié de l'expression subjective ordinaire. [...] c'est le lieu de rencontre interindividuel, qui peut donner lieu à un partage de valeurs si les croyances sont communes ou compatibles, ou à un affrontement dans le cas contraire [...].» (Caelen-Haumont 2002: 23) «[...] les mélismes apparaissent principalement dans les phases de recherche, notamment dans les reformulations qui ont une fonction principale de relance et de valorisation de la parole des enfants. Ils visent à manifester et à faire partager un intérêt [...]» (Vuillet, thèse sur les interactions en maternelle, cit. par Caelen-Haumont 2008: 172)

27. Dans des langues autres que le français pour ce qui est de la fonction distinctive.

28. Nous faisons référence à ce propos d'A. Culioli : («On peut désigner aussi par épilinguistique ce qui se passe à un moment d'énonciation dans la tête de l'interlocuteur ou du locuteur?») : «Non, je l'emploie comme, véritablement, une activité permanente dont nous n'avons pas 
conscience et qui nous fournit ses représentations qui s'entrecroisent, s'entrechoquent etc. et qui vont faire que vous avez parfois de ces sens!» (Normand 2012: 37)

\section{RÉSUMÉS}

L'article traite d'une courbe intonative qui accompagne la reprise d'un propos précédent, en contestant le bien-fondé de ce propos. Cette intonation construit à elle seule un commentaire critique sur l'adéquation du dit à bien représenter l'état de choses considéré comme vrai par le locuteur-énonciateur, elle est donc clairement de nature métalinguistique. En opposition avec d'autres intonations à valeur modale ou attitudinale, nous en analysons les caractéristiques formelles et sémantiques, en proposant de mettre en correspondance ces deux ordres de paramètres.

The purpose of this article is to present an intonative curve that accompanies the repetition of a previous statement, questioning the validity of this statement. This intonation itself constitutes a critical commentary on the adequacy of what is said to properly represent the state of things considered true by the speaker-enunciator, so it is clearly of a metalinguistic nature. In contrast to other intonations of modal or attitudinal value, we analyse its formal and semantic characteristics, proposing to relate these two orders of parameters.

\section{INDEX}

Mots-clés : intonation, sémantique énonciative, modalité

Keywords : intonation, semantics, enunciation, modality

\section{AUTEUR}

EVELYNE SAUNIER

Université de Paris Descartes - Laboratoire MoDyCo 